\title{
融合图像方法的半实时轴承故障诊断方法
}

\author{
王鹏志 ${ }^{1,2,3)}$ ，张满击 ${ }^{3,4)}$, 韩亚洪 ${ }^{1,2)^{*}}$, 赵旭 ${ }^{3)}$, 王征 ${ }^{1,2}$ ) \\ ${ }^{1)}$ (天津大学智能与计算学部 天津 300350) \\ 2) (天津市机器学习重点实验室 天津 300050) \\ 3) (河北工业大学人工智能与数据科学学院 天津 300401) \\ 4) (天津市虚拟现实与可视计算国际联合中心 天津 300401) \\ (yahong@tju.edu.cn)
}

\begin{abstract}
摘 要：轴承在高负荷的环境下长时间运转经常会发生故障, 造成巨大损失. 若能在故障发生前期进行准确感知, 则可以在很大程度上减少损失. 通过分析轴承故障问题的特点，提出一种半实时的高准确率诊断方法，使用双路宽 卷积核深度卷积网络(deep convolutional neural networks with double paths and wider kernels, DWDCNN)作为实时诊断 算法, 在结果出现异常或轴承处于高噪声环境下的时候对轴承的振动数据使用短时傅里叶变换(short time Fourier transform, STFT), 将其转换为图像, 再使用轻型多尺度胶囊网络(smaller inception capsule net, SICN)进行二次诊断. 使用该算法与现有其他算法在凯斯西储大学(Case Western Reserve University, CWRU)数据集和添加不同强度噪声后 的 CWRU 数据集上进行实验, 对准确性和计算效率进行对比. 结果显示 DWDCNN 模型使用 $0.12 \mathrm{~ms}$ 即可完成一次预 测, 且在 $S N R=-4 \mathrm{~dB}$ 的条件下达到 $80.07 \%$ 的准确率, 而 $\mathrm{SICN}$ 模型虽然计算时间较长, 但是可以在 $\mathrm{SNR}=-4 \mathrm{~dB}$ 的条 件下达到 $95.00 \%$ 的准确率.
\end{abstract}

关键词: 轴承: 故障诊断：振动信号：卷积神经网络

中图法分类号: TP391.41 DOI: 10.3724/SP.J.1089.2021.18579

\section{Semi-Real-Time Bearing Fault Diagnosis Method Combined Image Method}

\author{
Wang Pengzhi ${ }^{1,2,3)}$, Zhang Mandun ${ }^{3,4)}$, Han Yahong ${ }^{1,2)^{*}}$, Zhao $\mathrm{Xu}^{3)}$, and Wang Zheng ${ }^{1,2)}$ \\ 1) (College of Intelligence and Computing, Tianjin University, Tianjin 300350) \\ 2) (Tianjin Key Lab of Machine Learning, Tianjin 300050) \\ 3) (School of Artificial Intelligence, Hebei University of Technology, Tianjin 300401) \\ 4) (Tianjin International Joint Center for Virtual Reality and Visual Computing, Tianjin 300401)
}

\begin{abstract}
Bearings running in the high load environment for a long time often malfunction, resulting in huge losses. It can be reduced to a large extent if the fault can be detected accurately in the early stage. According to the analysis on characteristics of the bearing fault problem, a semi-real-time and high-accuracy diagnosis method is proposed. First, deep convolutional neural networks with double paths and wider kernels (DWDCNN) are used as a real-time diagnosis method. When the result looks abnormal or the bearing is in a high-noise environment, short-time Fourier transform (STFT) is used on the vibration data of the bearing to convert it to image, and smaller inception capsule net (SICN) is used for secondary diagnosis. Then a comparison experiment between the proposed models and other existing models on Case Western Reserve University (CWRU) dataset and CWRU
\end{abstract}

收稿日期：2020-08-16; 修回日期：2020-11-04. 基金项目：国家重点研发计划(2019YFC1520600); 国家自然科学基金(61573356); 河北省自然科学基金(F2019202054). 王鹏志(1996一), 男, 硕士研究生, 主要研究方向为基于机器学习的可靠性分析和故障诊断; 张满圈(1971-), 男, 博士, 副教授, 硕士生导师, 主要研究方向为计算机图形学、图像处理、三维模型检索、三维物体识别: 韩亚洪 (1980-), 男, 博士, 博士生导师, 论文通讯作者, 主要研究方向为人工智能、机器学习、态度感知与决策、自主学习; 赵旭(1998一), 男, 硕士研究生, 主要研究方向为基于深度学习的图像处理、三维模型数据处理; 王征(1980-), 男, 博士, 副教授, 硕士生导师, 主 要研究方向为人工智能、图像处理与计算机视觉、时序数据显著性识别. 
dataset with different intensities of noise is made on the aspects of accuracy and time performance. The result shows that DWDCNN can accomplish one prediction within $0.12 \mathrm{~ms}$, and the accuracy can be achieved of $80.07 \%$ under the condition of SNR $=-4 \mathrm{~dB}$. Although using more time, the accuracy of SICN can be achieved of $95.00 \%$ under the condition of $\mathrm{SNR}=-4 \mathrm{~dB}$.

Key words: rolling bear; fault diagnosis; vibration signal; convolutional neural networks

据统计，发生在旋转机械的故障中约有 $70 \%$ 的 故障与轴承有所关联, 并且轴承一旦出现损伤, 势 必引发一系列连锁故障, 最终有可能导致整个设备 的痽疾. 此外, 在《中国制造 $2025 》$ 计划中，对机 械设备健康状况以及故障的检测已被列为智能制造 中的核心技术 ${ }^{[1]}$. 因此, 对轴承状态的检测或故障 诊断研究的重要性可想而知. 针对轴承故障诊断问 题, 有以下几种常见的基于深度学习的解决算法.

（1）基于深度置信网络(deep belief networks, DBN)的故障诊断算法. 早在 2013 年, Tamilselvan 等 ${ }^{[2]}$ 就提出基于健康状态分类的 $\mathrm{DBN}$ 故障检测算 法，并与基于支持向量机 ${ }^{[3]}$ (support vector machine, SVM)的检测算法进行了比较, 说明了该算 法的有效性. 在其后几年中, DBN 经常配合其他技 术一同解决故障检测问题. 例如, Shao 等 ${ }^{[4]}$ 提出一 种基于自适应 DBN 和双树复小波包(dual-tree complex wavelet packet, DTCWPT)相结合的诊断 算法, 取得了更优秀的性能.

(2) 基于自动编码器(autoencoder, AE)的故障 诊断算法. $\mathrm{Lu}$ 等 ${ }^{[5]}$ 设计了一种由 3 个堆叠自编码器 (stacked autoencoder, SAE)组成的堆叠去噪 $\mathrm{AE}$, 对 原始的凯斯西储大学(Case Western Reserve University, CWRU)数据集施加 $15 \mathrm{~dB}$ 的随机噪声, 并 使用多工况数据集作为测试集，达到了最低 $91.79 \%$ 的准确率，比传统的 SAE 算法提高了 $3 \% \sim 10 \%$. Guo 等 ${ }^{[6]}$ 用了另一种形式的 SAE 将 CWRU 数据集的时序数据处理为频域信号, 其结 果比 DBN 算法具有更低的误差, 尤其在加噪的情 况下，其准确率比 DBN 提高了约 7\%. 2019 年, Hung Nguyen 等 ${ }^{[7]}$ 提出了一种由 SAE 和 Softmax 分 类器构成的深度学习网络, 首先通过集合经验模态 分 解 (ensemble empirical mode decomposition, EEMD)进行预处理, 再输人到网络中进行训练.

(3) 基于循环神经网络(recurrent neural networks, RNN)的故障诊断算法. 最早在 2005 年出现 了将 RNN 用于故障诊断的算法，但由于梯度爆炸 或梯度消失等问题，该算法在相当一段时间内没 有新发展. 直到 2015 年, Abed 等 ${ }^{[8]}$ 将 RNN 用于轴
承故障诊断，没有使用端到端的检测，而是通过离 散小波变换将时序数据变换为频域数据再输人网 络中进行分类. Pan 等 ${ }^{[9]}$ 在 2018 年提出了一种将一 维卷积神经网络 (convolution neural networks, $\mathrm{CNN}$ ) 与长短期记忆 (long short-term memory, LSTM)单元相结合进行轴承故障分类的算法. 该 算法将一维时序信号作为输人, 通过一维卷积层 与最大值池化层, 再使用 LSTM 层接上全连接层完 成分类, 搭建了加上输人输出层一共 5 层的网络, 最终达到了接近 $100 \%$ 的准确率.

（4）基于 CNN 的故障诊断算法. 自从 2016 年 $\mathrm{CNN}$ 被首次用于识别轴承故障以来, 出现了很多 基于该类网络模型的研究，从对数据的预处理、模 型的抗噪能力、训练速度和参数的数量等多方面不 断改善 $\mathrm{CNN}$ 的故障检测能力与泛化能力. 其中, 一维 $\mathrm{CNN}$ 模型由于层结构比较固定, 相对简单, 参数较少, 以及训练速度和准确率相对较高的优 势, 得到了非常广泛的应用. 其后, 出现了许多将 $\mathrm{CNN}$ 与其他算法结合用于轴承故障检测的算法. Qian 等 ${ }^{[10]}$ 使用自适应交叠 CNN(adaptive overlapping convolutional neural networks, AOCNN), 直接 处理一维振动信号, 并且在自适应卷积层将样本 分割成若干部分，再采用稀疏滤波获取局部特征， 最终可以在轴承健康情况分为 10 类的情况下达到 $99.19 \%$ 的准确率. Hoang 等 ${ }^{[11]}$ 则通过将原始的一维 信号转换为二维图像, 并利用 $\mathrm{CNN}$ 在图像分类上 的优势, 最终可使模型在 CWRU 数据集上达到将 近 $100 \%$ 的准确率. 此外, 在不重新训练的情况下, 该算法仍能在加噪后的数据上获得较高的准确率, 显示出较强的抗噪声能力.

从上述算法可以看出, 大部分算法均可以达 到较高的准确率. 但对于非端到端的识别算法均 存在抗噪能力较弱或处理时间过长等问题, 如使 用 EEMD 作为预处理, 虽然对噪声的抵抗能力较 强, 但需要耗费相当长的时间, 无法对轴承做到实 时的故障诊断. 而基于 RNN 的算法，如 LSTM 网络 等需要大量的训练集和训练时间才能达到较高的准 确度, 且其泛化能力普遍较差. 传统的二维 $\mathrm{CNN}$ 需 
要提取图像特征及较多的样本数量, 且训练出的模 型较大, 若希望进行实时诊断, 则需要运算能力非 常强的图形处理器 (graphics processing unit, GPU).

综上所述，本文提出一种半实时的轴承故障 诊断方法. 由于在实际情况中通常需要对轴承进 行实时的故障诊断，但具有高实时性的诊断模型 参数较少, 在非理想情况下诊断的准确率相对较 低. 因此, 本文使用基于张伟 ${ }^{[12]}$ 提出的宽卷积核 深度卷积网络(deep convolutional neural networks with wide first-layer kernel, WDCNN)的改进算法双 路宽卷积核深度卷积网络 (deep convolutional neural networks with double paths and wider kernels, DWDCNN)作为实时的基础诊断算法，并且使用基 于图像的 SICN(smaller inception capsule net)作为 定期诊断或异常情况下的诊断算法.

\section{1 本文方法}

由于轴承故障通常对实时性有着较高的要求, 因此往往要求预测模型较小. 而较小的模型通常 存在对训练数据的欠拟合或泛化能力较差的问题, 导致最终的准确率偏低. 将时序数据图像化的方 法虽然准确度非常高，但欠缺实时性. 因此，本文 提出图 1 所示半实时的轴承故障诊断方法.

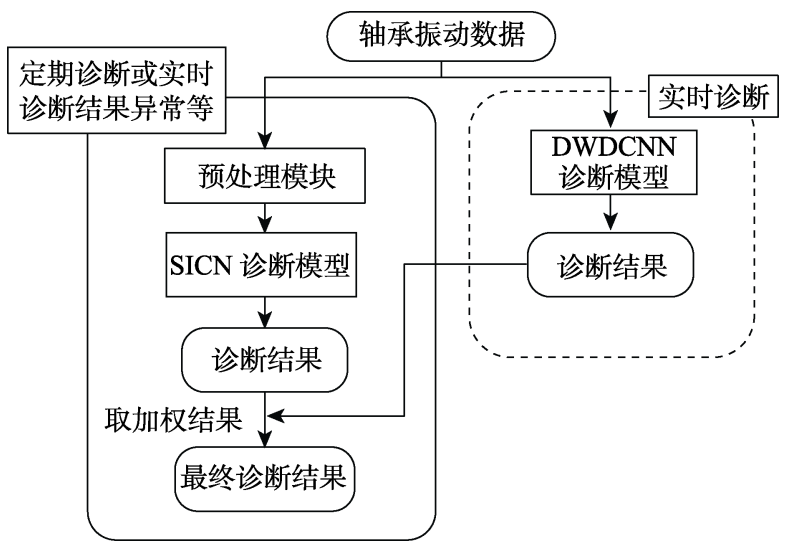

图 1 本文方法结构

本文提出的基础诊断网络 DWDCNN 参数相 对较少, 可以快速地对采样得到的振动数据分析, 且经测试该算法在大多数环境下准确率相对较高, 可以应对一些有干扰的环境. 而对于有较多干扰 的环境或 DWDCNN 诊断出的结果飘忽不定的情 况, 再使用本文基于图像的高准确率诊断网络 SICN 进行二次诊断，其诊断结果的权重值高于 DWDCNN. SICN 算法虽然比原网络有所精简，但 运算时间仍相对较长，难以在成本相对较低的情
况下达到实时的要求，因此最后取一段时间内诊 断结果的加权最高者作为最终的诊断结果.

\section{1 基础诊断方法}

\subsubsection{DWDCNN 模型}

本文提出的 DWDCNN 模型网络结构如图 2 所 示. DWDCNN 在 WDCNN 的基础上，首先减小第 1 个卷积层中的卷积步长, 再并联上一条与其类似 的网络结构. 针对同一输人, 将其送人 2 个大分支 中, 其中包含 WDCNN 结构的分支中又包含一条 小分支, 并在第 3 次卷积操作后进行特征融合, 在 进行 2 次卷积操作后再与另一条大分支进行特征 融合. 为方便表达, 下文中将图 2 中的 Path1 称作 路径 1, Path2 称作路径 2. 每一层中的具体参数如 表 1 所示. 表 1 中， $s$ 代表步长, 若在卷积参数中则 代表卷积步长, 若在池化参数中则代表池化步长, 下文中若出现此类情况则意义相同.

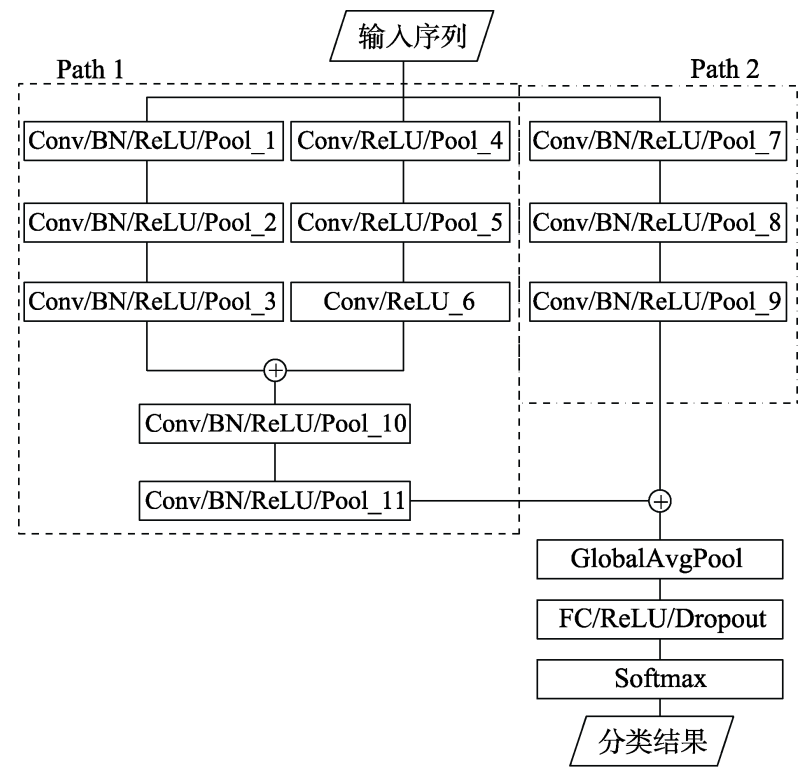

图 2 DWDCNN 模型结构

表 1 DWDCNN 模型结构的各层参数

\begin{tabular}{lrc}
\multicolumn{1}{c}{ 网络层 } & 卷积参数 & 池化参数 \\
\hline Conv/BN/ReLU/Pool_1 & $84 \times 1 s 6,16$ & $2 \times 1 s 2$ \\
Conv/BN/ReLU/Pool_2 & $3 \times 1 s 1,32$ & $2 \times 1 s 2$ \\
Conv/BN/ReLU/Pool_3 & $3 \times 1 s 1,32$ & $2 \times 1 s 2$ \\
Conv/ReLU/Pool_4 & $32 \times 1 s 3,32$ & $2 \times 1 s 2$ \\
Conv/ReLU/Pool_5 & $16 \times 1 s 2,32$ & $2 \times 1 s 2$ \\
Conv/ReLU/Pool_6 & $8 \times 1 s 2,32$ & $4 \times 4 s 4$ \\
Conv/BN/ReLU/Pool_7 & $32 \times 1 s 3,32$ & $2 \times 1 s 2$ \\
Conv/BN/ReLU/Pool_8 & $16 \times 1 s 2,32$ & $2 \times 1 s 2$ \\
Conv/BN/ReLU_9 & $8 \times 1 s 2,32$ & \\
Conv/BN/ReLU/Pool_10 & $3 \times 1 s 1,32$ & $2 \times 1 s 2$ \\
Conv/BN/ReLU/Pool_11 & $3 \times 1 s 1,32$ & $2 \times 1 s 2$ \\
GlobalAvgPool & & \\
FC/ReLU/DropOut & & \\
Softmax & & \\
\hline
\end{tabular}




\subsection{2 对 WDCNN 模型的参数改进}

由于 DWDCNN 模型仍基于 WDCNN, 因此除 使用特征融合加强模型性能外，还将改进 WDCNN 的模型参数.

因为轴承的振动信号实际上是具有周期性的, 且记录起点的相位值完全随机的，为使 DWDCNN 可以更好地学习到与初始相位无关的特征，全连 接层前 1 层中的神经元对应原始输人中的区域长 度不应与实际周期过于接近，且最好大于一个周 期，以获得更稳定的特征和更加随机的起点. 若全 连接层前 1 层的神经元对应原始输人的区域长度 为 $a^{\{0\}}, T$ 为轴承旋转 $360^{\circ}$ 所对应的数据长度， $L$ 为所输人时序数据的长度, 则应有关系式 $T<a^{\{0\}} \leqslant L$. 则第 $l$ 个池化层中的神经元对应前 1 个池化层中区域长度的关系式为

$$
a^{\{l-1\}}=s^{\{l\}}\left(p^{\{l\}} a^{\{l\}}-1\right)+w^{\{l\}}
$$

其中, $S^{\{l\}}$ 代表第 $l$ 个卷积层的卷积步长; $p^{\{l\}}$ 代表 第 $l$ 个池化层的池化窗口步长; $w^{\{l\}}$ 代表第 $l$ 个卷 积层的卷积核长度，其中由于仅有边缘的神经元 对应到填补区域，故忽略填补的影响.

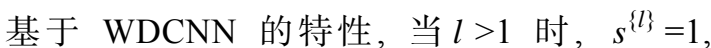
$w^{\{l\}}=3, \quad p^{\{l\}}=2$ : 式(1)可简化为

$$
a^{\{l-1\}}=2\left(a^{\{l\}}+1\right)
$$

因此，若有 5 层卷积层，则

$$
a^{\{0\}}=s^{\{1\}}\left(2\left(16 a^{\{5\}}+30\right)-1\right)+w^{\{1\}}
$$

与文献[12]不同，由于文献[12]中的输人信号 长度锁定为 2048 , 且信号长度应该被步长整除, 因此在上述推导后, 锁定为 8 或 16 . 本文中的输人 信号长度将取拥有更多质因数的数值, 以此来改 变步长, 进而探索更优的模型结构.

\section{2 基于图像的诊断方法}

\section{2 .1 胶囊网络}

由于轴承的工作环境往往并不固定，又因为 无法测量或记录所有条件下的数据，因此在环境 因素经常变化的条件下，故障诊断算法几乎很难 保证诊断结果的准确性. 解决该问题的关键是提 取已有数据中最本质上的特征. 在提取特征的算 法中，目前最常见的便是直接使用 $\mathrm{CNN}$.

对于传统 $\mathrm{CNN}$, 池化层几乎可以说是必不可 少的构成元素. 池化层首先是个很强的非线性层, 对输人进行了一个非线性很强的变换，更有利于 拟合; 池化层本身没有参数，还可以减少非常多的 特征输出, 使模型包含的参数大幅度减少, 并且降 低了模型过拟合的风险。但由于池化层在减少特
征的同时，也会造成对变化的不敏感，因此降低了 域自适应能力.

图 3 所示为 4 种不同故障轴承的振动序列使用 短时傅里叶变换 (short time Fourer transform, STFT)后的结果. 由图 3 可见, 这些图像虽然并不 复杂, 但十分抽象, 因此提取到最本质的特征对某 些极端条件下分类结果的准确性十分关键. 与基于 图像的算法相比, 简单的一维 $\mathrm{CNN}$ 难以提取到这 些特征，仅仅是寻找振动序列中的高频区域 ${ }^{[12]}$; 而 当出现较大的噪声干扰时, 其准确率将明显下降.
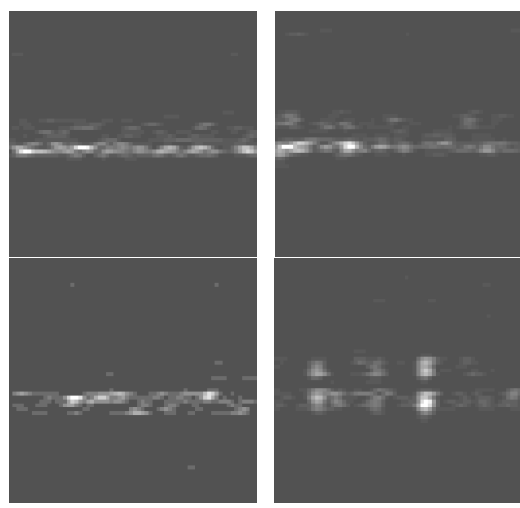

图 3 STFT 变换结果示例

此外, 又因为针对轴承的故障诊断需要一定 的实时性，因此也不适宜使用过深的网络模型. Sabour 等 ${ }^{[13]}$ 提出的胶囊网络正好可以解决上述问 题. 本文提出的网络从胶囊网络出发加以适当改 进, 利用该网络无池化层的特点, 减少在某些特征 细节上的丢失，使其更加适应轴承故障的诊断.

(1) Squash 函数

由于胶囊网络特殊的输出，即一个胶囊向量 成为特征表达的最小单位, 也使传统激活函数已 不再适用, 取而代之的是新的 Squash 函数, 其公 式为

$$
\boldsymbol{c}_{o}^{j}=\operatorname{Squash}\left(\boldsymbol{c}_{i}^{j}\right)=\frac{\left\|\boldsymbol{c}_{i}^{j}\right\|^{2}}{1+\left\|\boldsymbol{c}_{i}^{j}\right\|^{2}} \cdot \frac{\boldsymbol{c}_{i}^{j}}{\left\|\boldsymbol{c}_{i}^{j}\right\|^{2}}
$$

其中, $\boldsymbol{c}_{i}^{j}$ 为输人的第 $j$ 个胶囊向量; $\boldsymbol{c}_{o}^{j}$ 为输出的 第 $j$ 个胶囊向量. Squash 含义为挤压, 旨在将胶囊 向量的模长挤压至 $[0,1)$ 内, 在某种程度上相当于 归一化操作.

(2) 动态路由算法

在胶囊网络中, 还引人了类似聚类的动态路 由算法. 由于经过训练后, 所有参数均已固定, 而 使用这种动态路由算法, 除了可以在一定程度上 调整拟合的程度, 还可以提高模型的泛化能力. 算 
法步骤如下所示, 其中 $R$ 代表动态路由次数.

输人. 第 $l$ 层中的所有胶囊及其输出 $\hat{\boldsymbol{u}}$, 以及 路由迭代计数 $r$.

输出. 高层胶囊 $\boldsymbol{v}_{j}$.

Step1. 对所有在 $l$ 层中的胶囊 $i$, 和在第 $l+1$ 层的 胶囊 $j$, 初始化中间量 $b_{i j}=0$.

Step2. 根据 $r$ 迭代计算 $\boldsymbol{v}_{j}$ :

Step2.1. 对所有在 $l$ 层中的胶囊 $i$, 初始化变量 $c_{i j}=\exp \left(b_{i j}\right) / \sum_{k} \exp \left(b_{i k}\right) ;$

Step2.2. 对所有在 $l+1$ 层中的胶囊 $j$, 初始化原 始胶囊向量 $\boldsymbol{s}_{j}=\sum c_{i j} \hat{\boldsymbol{u}}_{j}$;

Step2.3. 对所有在 $l+1$ 层中的原始胶囊 $j$, 初始 化其激活后的向量 $\boldsymbol{v}_{j}=\operatorname{Squash}\left(\boldsymbol{s}_{j}\right)$;

Step2.4. 对所有在 $l$ 层中的胶囊 $i$ 和在第 $l+1$ 层 的胶囊 $j$, 计算 $b_{i j}=b_{i j}+\hat{\boldsymbol{u}}_{j} \cdot \boldsymbol{v}_{j}$.

(3) 损失函数

由于胶囊网络包含 1 个输人与 2 个输出, 使其 包含 2 种损失函数, 分别是计算输出类别与目标类 别损失的 Margin 损失函数, 以及重构输出与原输 出损失的 MSE(mean square error)损失函数. Margin 损失函数和 MSE 损失函数分别为

$$
\begin{aligned}
L_{k}= & T_{k} \max \left(0, m^{+}-\left\|\boldsymbol{v}_{k}\right\|\right)^{2}+ \\
& \lambda\left(1-T_{k}\right) \max \left(0,\left\|\boldsymbol{v}_{k}\right\|-m^{-}\right)^{2} \\
L_{\mathrm{MSE}} & =\frac{1}{N} \sum_{i=1}^{N}\left(y_{i}-\hat{y}_{i}\right)^{2}
\end{aligned}
$$

式(5)中, 如果分类 $k$ 出现, 则 $T_{k}=1$; 否则 $T_{k}=0$; 文献[13]中, $m^{+}=0.9, m^{-}=0.1, \lambda=0.5$. 因此, 胶囊 网络的最终损失函数为

$$
L_{\text {total }}=L_{k}+k \cdot L_{\mathrm{MSE}}
$$

其中, $k$ 为权重系数, 由于 Margin 损失函数与输 出结果最为密切, 故通常 $k<0.5$.

\subsection{2 数据预处理}

由于时域谱看起来杂乱无章, 并且存在过多 细节，不容易提取到本质的特征，因此本文模型将 时域转化至频域, 这样更便于提取到本质的特征, 更容易让神经网络拟合. 由于滚动轴承的振动信 号属于非平稳信号, 故采用 STFT. 其思想是假设 待分析信号在一段时间内可视作平稳的，再使用 大小确定的窗函数对待分析信号进行分段卷积. 为方便计算机处理，将其离散化为

$$
X(m, \omega)=\sum_{n=-\infty}^{\infty} x[n] w[n-m] \mathrm{e}^{-\mathrm{j} \omega n}
$$

其中, $x[n]$ 为待分析信号在时间 $n$ 时刻时的幅值: $w[n]$ 为窗函数, 在该数据预处理中使用的是汉宁 函数, 其数学公式为

$$
w[n]=\frac{1}{2}\left[1+\cos \left(\frac{2 \pi n}{N-1}\right)\right]
$$

经过 STFT, 可以将原始的一维时序信号转为 二维的频域图. 但由于转换后的二维数组的值相 对较小, 均趋近于 0 , 因此将其放大为原来的几十 倍或几百倍, 再送人网络. 本文将 STFT 后的结果 放大为原来的 400 倍再输人.

\subsubsection{SICN 模型}

本文提出的 SICN 模型基于 $\mathrm{ICN}^{[14]}$, 主要针对 原模型中参数冗余的问题进行改进, 其结构如图 4 所示. 其中第 3 次卷积类似 Inception ${ }^{[15]}$ 结构, 而胶 囊层所做的实际上是动态路由算法, 本文中参数 $r=3$, 在下一层中取该层结果中模长最大向量的 对应下标作为分类结果, 对其进行独热编码后再 与胶囊层的结果相乘，获得将非预测类别向量“遮 盖”后的结果, 送人后续的全连接层, 重构出输人 图像. 将输人图像与重构图像的均方差作为损失 函数的一部分，进而提升诊断结果的准确率.

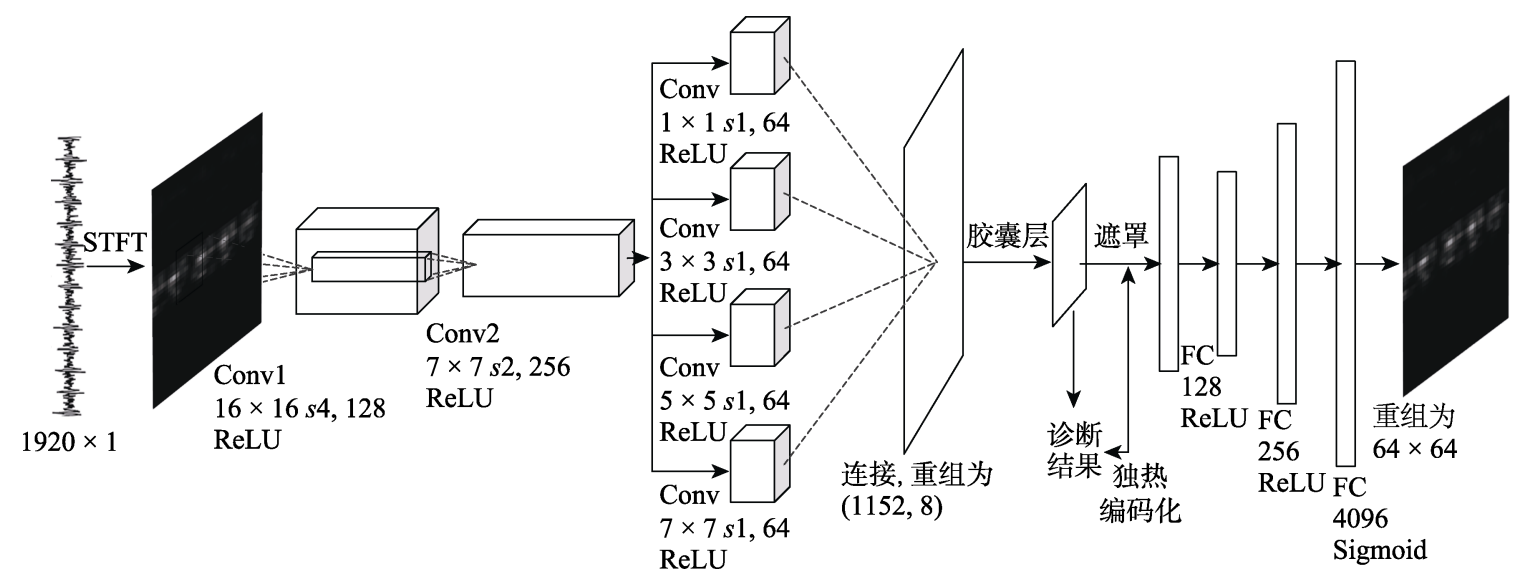

图 4 SICN 模型结构 


\section{2 实验及结果分析}

分别使用本文提出的 DWDCNN 与 SICN 进行 训练, 并对其准确率与时间性能等进行评估.

\section{1 数据集}

针对基于深度学习的轴承故障检测选择了在 该领域使用最广泛的 CWRU 数据集. 官方网站提 供的数据格式为.mat. 实验所用轴承型号为较为常 见的 SKF 6205-2RSJEM.

该数据集分别由 4 个部分构成: 健康轴承振动 数据、 $12000 \mathrm{~Hz}$ 采样率的驱动端故障轴承振动数 据、 $48000 \mathrm{~Hz}$ 采样率的驱动端故障轴承振动数据 与风扇端故障轴承数据. 本文使用应用最多的 $12000 \mathrm{~Hz}$ 采样率的驱动端故障轴承振动数据. 其 中轴承在运作时又分为 4 种转速: 1797 round $/ \mathrm{min}$, $1772 \mathrm{round} / \mathrm{min}, 1750 \mathrm{round} / \mathrm{min}, 1730 \mathrm{round} / \mathrm{min}$, 分别对应 4 种负载: $0 \mathrm{~W}, 745.700 \mathrm{~W}, 1491.400 \mathrm{~W}$, $2234.100 \mathrm{~W}$.

轴承的故障种类共有 3 大类, 分别为内圈故 障、滚珠体故障与外圈故障. 此外，每种故障又分 为不同的损伤直径, 如 $0.1778 \mathrm{~mm}, 0.3556 \mathrm{~mm}$.

为验证模型对加噪数据预测结果的可靠性, 本文使用高斯白噪声对 CWRU 数据集进行不同程 度的加噪, 并使用信噪比(signal noise ratio, SNR) 作为衡量噪声强度的标准, 如当 $\mathrm{SNR}=0 \mathrm{~dB}$ 时, 说 明噪声幅值或功率与源信号相当.

\subsection{DWDCNN 模型}

\subsection{1 训练环境以及超参数设置}

该模型在服务器中完成训练, 使用 Python 语 言编写，深度学习框架为 Pytorch, DWDCNN 的训 练超参数设置如表 2 所示.

表 2 DWDCNN 训练超参数设置

\begin{tabular}{ll}
\hline \multicolumn{1}{c}{ 超参数 } & \multicolumn{1}{c}{ 取值 } \\
\hline 迭代数 & 200 \\
学习率 & 0.001 \\
学习率递减系数 & 0.999 \\
批次大小 & 32 \\
优化器 & Adam 优化器 \\
\hline
\end{tabular}

\subsection{2 实验方法}

为进一步加强 WDCNN 的性能, 本文测试了 当第 1 层卷积步长从 4 16 对应的最终准确率, 其 中当步长为 16 时即为原始的 WDCNN. 结果表明 当第 1 层卷积步长为 6 时效果最佳, 为方便表述, 以下将第 1 层卷积步长为 6 的模型称为短步长宽卷
积核深度卷积网络 (deep convolutional neural networks with shorter strides and wider kernels, WSDCNN). 另外, 针对故障检测问题, 不可仅注 重加噪验证集上的准确率，更应该重视未加噪验 证集的准确率，故定义加权准确率，再由每次最高 加权准确率对应的加噪验证集的准确率求平均值 得出, 其数学表达式为

$$
\frac{1}{m} \sum_{i=1}^{m} \underset{a^{\mathrm{n}}}{\operatorname{argmax}}\left(w_{1} a_{(i, j)}+w_{2} a_{(i, j)}^{\mathrm{n}}\right) .
$$

其中, $0<j \leqslant E, E$ 代表所需要的迭代次数; $a^{\mathrm{n}}$ 代表加噪验证集准确率; $w_{1}=0.9, w_{2}=0.1$.

WSDCNN 与 WDCNN 的对比如表 3 所示. 可 见, 在各个情况下 WSDCNN 的准确率均优于原来 的 WDCNN.

表 32 种模型平均加权准确率对比

\begin{tabular}{ccc}
\hline 噪声强度 $/ \mathrm{dB}$ & WDCNN $(s=16)$ & WSDCNN $(s=6)$ \\
\hline-4 & 66.44 & $\mathbf{7 0 . 0 1}$ \\
-2 & 82.54 & $\mathbf{8 4 . 3 7}$ \\
0 & 90.60 & $\mathbf{9 2 . 6 6}$ \\
2 & 96.01 & $\mathbf{9 7 . 3 0}$ \\
4 & 97.89 & $\mathbf{9 8 . 9 5}$ \\
6 & 99.07 & $\mathbf{9 9 . 7 4}$ \\
8 & 99.18 & $\mathbf{9 9 . 7 7}$ \\
10 & 99.26 & $\mathbf{9 9 . 8 4}$ \\
\hline 平均 & 91.37 & $\mathbf{9 2 . 8 3}$ \\
\hline
\end{tabular}

注. 粗体表示最优结果.

常见的特征融合方法共有 2 种，一种为使用 concatenate 方法将多条支路中的通道组合起来, Inception 结构正是使用的该结构; 另一种则是使 用加法将多条支路中的对应通道进行加和, 如 FPN(feature pyramid networks) ${ }^{[16]}$ 中使用的特征融 合方法正是加法. 由于使用 concatenate 方法后, 模 型的训练结果出现极不稳定的现象, 因此选用加 法进行特征融合操作.

由于改进后的网络比原 WDCNN 多出了 2 条 支路, 为验证这 2 条支路的有效性, 分别将 2 条支 路与减小第 1 个卷积层步长后的 WSDCNN 组合, 再与不加支路和使用全部支路的 DWDCNN 进行 对比, 探究这 2 次特征融合对模型性能的影响情 况. 对比结果如表 4 所示. 其中 P1 代表模型中路 径 1 的右侧分支, P2 则代表路径 2 分支.

由表 4 可以看出，当仅使用 $\mathrm{WSDCNN}+\mathrm{P} 1$ 时， 在较高信噪比的情况下, 其准确率相比 WSDCNN 
乃至改进后的 DWDCNN 均稍高一些; 而仅使用 $\mathrm{WSDCNN}+\mathrm{P} 2$ 时, 则在较低信噪比的情况下准确 率较高, 在信噪比为 $-4 \mathrm{~dB}$ 与 $-2 \mathrm{~dB}$ 的情况下尤为 明显, 相比 WSDCNN 高出了 10. 说明 P2 可以使 该算法获得较大的抗噪能力上的提升，但在高信 噪比时, 其准确率相比 WSDCNN 或 WSDCNN+P1 稍低. 而 DWDCNN 则更加均衡, 在低信噪比情况 下表现出了 WSDCNN+P2 的特点, 高信噪比时的 准确率则在 WSDCNN+P1 与 WSDCNN+P2 之间, 最终在各种情况下的平均准确率最高.

表 4 不同分支组合在不同噪声下对准确率的影响 \%

\begin{tabular}{ccccc}
\hline 噪声强度/dB & WSDCNN & \multicolumn{4}{l}{ WSDCNN+P1 } & WSDCNN+P2 DWDCNN \\
\hline-4 & 70.01 & 73.08 & $\mathbf{8 0 . 4 1}$ & 80.07 \\
-2 & 84.37 & 86.20 & 90.13 & $\mathbf{9 0 . 5 2}$ \\
0 & 92.66 & 96.10 & 96.87 & $\mathbf{9 7 . 1 7}$ \\
2 & 97.30 & 98.84 & $\mathbf{9 9 . 2 3}$ & 99.01 \\
4 & 98.95 & 99.46 & 99.16 & $\mathbf{9 9 . 5 3}$ \\
6 & 99.74 & $\mathbf{9 9 . 7 9}$ & 99.67 & 99.77 \\
8 & 99.77 & $\mathbf{9 9 . 9 1}$ & 99.70 & 99.83 \\
10 & 99.84 & $\mathbf{9 9 . 9 2}$ & 99.76 & 99.86 \\
\hline 平均 & 92.83 & 94.16 & 95.62 & $\mathbf{9 5 . 7 2}$ \\
\hline
\end{tabular}

注. 粗体表示最优结果.

如表 4 所示, 即便是使用 DWDCNN, 在噪声 较大的情况下, 如 $-4 \mathrm{~dB}$ 和 $-2 \mathrm{~dB}$, 其准确率依然不 够高. 可见，仅使用该网络无法满足某些极端条件 下的高准确率分类.

\subsection{SICN 模型}

\subsection{1 训练环境以及超参数设置}

该模型在服务器中完成训练, 使用 Python 语 言编写, 深度学习框架为 Pytorch, SICN 的训练超 参数设置如表 5 所示.

表 5 SICN 训练超参数设置

\begin{tabular}{ll}
\hline 超参数 & 取值 \\
\hline 迭代数 & 200 \\
学习率 & 0.00125 \\
学习率递减系数 & 0.999 \\
批次大小 & 32 \\
动态路由迭代次数 & 3 \\
$k$ 值 & 0.275 \\
优化器 & Adam 优化器 \\
\hline
\end{tabular}

经验证，当动态路由迭代次数为 3 时，时间性 能与准确率达到最佳平衡: 当取 $=0.275$ 附近的值时 效果最; $k$ 过大会导致过于注重重构部分的损失,
分类准确率不佳; $k$ 过小则会使重构部分失去意 义，同样导致准确率下降.

\subsection{2 数据预处理算法的选择}

将轴承的振动时序数据转换为图像的常见预 处理算法主要有 3 种: 直接转换为灰度图、使用离 散小波变换将时域数据转换为频域数据、快速傅里 叶变换(fast fourier transform, FFT) 将时域数据转换 为频域数据.

相比后 2 种算法, 使用直接转换为灰度图的算 法得到的图像显然更加难以分类，由于其本质上 仍是时序数据, 因此该算法的抗噪能力也相对较 弱. 而对于离散小波变换，实验发现使用该算法的 损失函数值下降速度明显慢于 FFT, 且最终的性能 同样不及使用 FFT 的结果. 因此, 本文使用 FFT 作 为预处理算法.

\subsubsection{SICN 的重构结果}

SICN 并非只输出对应轴承数据的健康状况类 别, 还有每种健康状况的重构图像. 经过多次训 练, 该算法所输出的每种类别的重构图像如图 5 所 示. 其中每对图像, 左侧为输人图像, 右侧为输出 的重构图像, 10 对图像分别对应轴承的 10 种健康 状态.
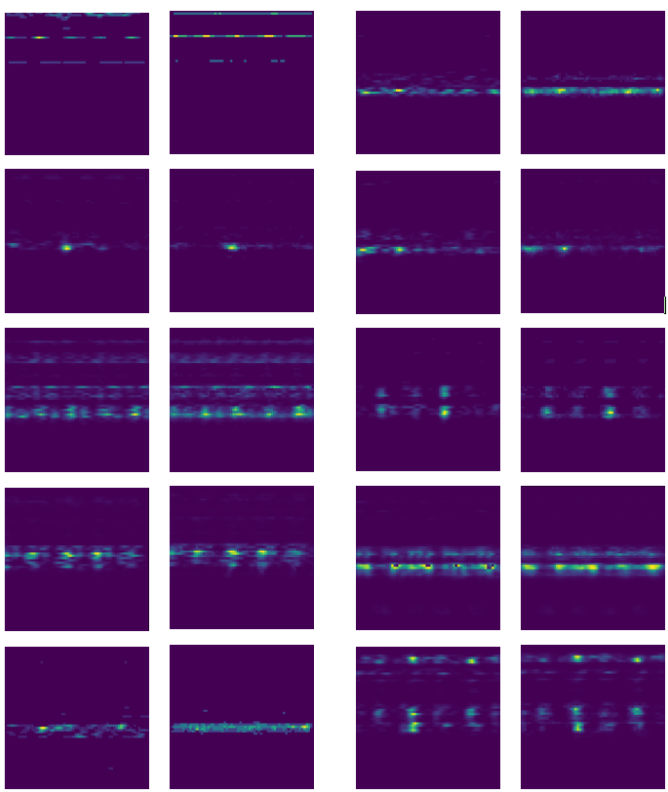

图 $5 \mathrm{SICN}$ 重构图与输人图的对比

从图 5 可以看出, SICN 针对轴承的 10 种健康 状态, 可以根据预测出的类别以及胶囊向量较好 地重现出最初输人到网络中的图像, 有些甚至难 以轻易看出区别. 由此可知, 胶囊网络已经学习到 了输人的 10 种健康状态所对应图像的特征及其各 
种变换，进一步降低了分类错误的可能性.

\section{4 与其他算法在不同噪声强度下的对比}

本节将本文提出的 DWDCNN, SICN 与其他算 法进行比较, 包括传统机器学习算法, 如 FFT+支 持向量机 $(\mathrm{FFT}+\mathrm{SVM}), \mathrm{FFT}+K$ 最近邻法 $(\mathrm{FFT}+$ $K \mathrm{NN}), \mathrm{FFT}+$ 随机森林法 $(\mathrm{FFT}+\mathrm{RF})$, 以及深度学习 算法，如抗噪卷积诊断网络 ${ }^{[17]}$ (convolutional neural network diagnosis model with anti-noise, ACNNDM), AlexNet ${ }^{[18]}$, WDCNN, WSDCNN 和基于训练干扰 的卷积神经网络 (convolution neural network with training interference, TICNN $)^{[12]}$. 比较这些算法在 SNR 从 $-4 \mathrm{~dB}$ 到 $10 \mathrm{~dB}$ 以及不加噪情况下的准确率. 其中 AlexNet 由于模型过大, 所以对模型中每层的 参数量做了不同程度的减小. 测试中, 每种条件依 然取加权准确率最高者对应的加噪验证集准确率 的 10 次平均值.

最终实验结果如图 6 所示, 其中 INF 代表不加 噪. 可以看出, 传统机器学习算法虽在不加噪的情 况下准确率较高, 但其在加噪情况下的准确率相 当之低，最高不及 $40.00 \%$, 说明在同样条件下这 些算法的泛化能力较差. ACNNDM 虽在加噪情况 下的准确率均优于传统机器学习算法, 但由于该 模型参数过少, 在不加噪情况下准确率过低, 说明 该模型未能完全拟合. 虽然 AlexNet 在某些情况下 的准确率比 WSDCNN 稍高, 但总体仍不如 WSDCNN 的准确率高.

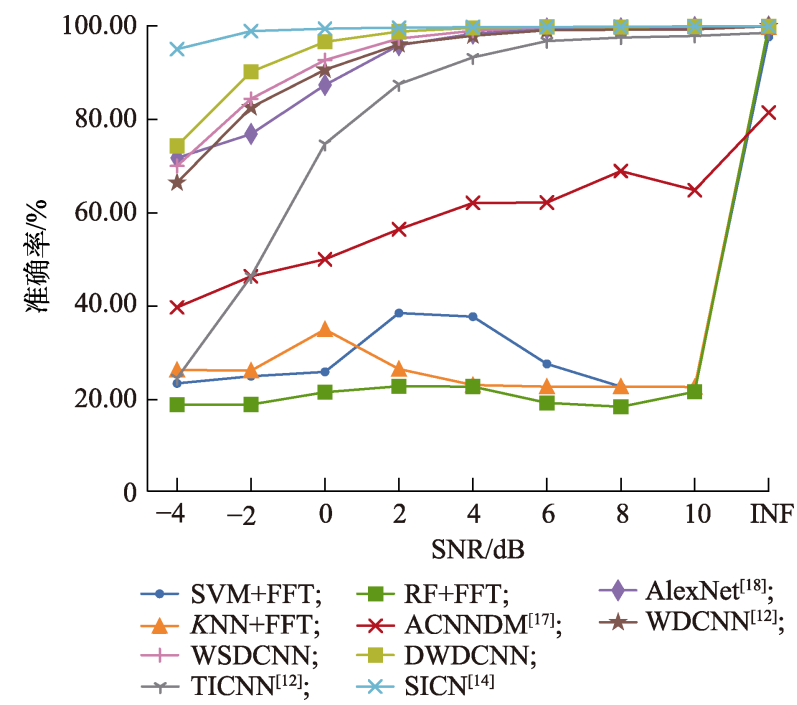

图 6 不同算法在不同噪声强度验证集的准确率对比

本文所提出的 DWDCNN 模型可以在保证模 型参数数量较小的前提下，在所有情况下的准确 率均优于 WSDCNN 和 WDCNN. 而基于图像方法 的 SICN 即便是在极端情况下，准确率也均明显优
于其他算法, 并且在大多数情况下的准确率趋于 $100 \%$, 即便在 SNR $=-4 \mathrm{~dB}$ 的情况下依然可以达到 $95.00 \%$ 的准确率，说明该模型具有更好的泛化能 力，可以应对噪声强度更大的场景.

\section{5 与其他模型在时间性能上的对比}

本节实验中所使用的深度学习框架为 Pytorch 1.3.1, 所使用计算机的 GPU 为 Tesla K80, 其显存 为 $12 \mathrm{~GB}$, 计算机内存为 $16 \mathrm{~GB}$.

本节使用上面提到的各个算法在各个噪声强 度情况下准确率的平均值(除去无噪声情况)以及 这些算法在预测过程中对一条长度为 1920 的振动 数据的平均时间消耗作为对比依据. 最终对比结 果如图 7 所示, 其中准确率越高, 时间消耗越低, 算法性能越好。

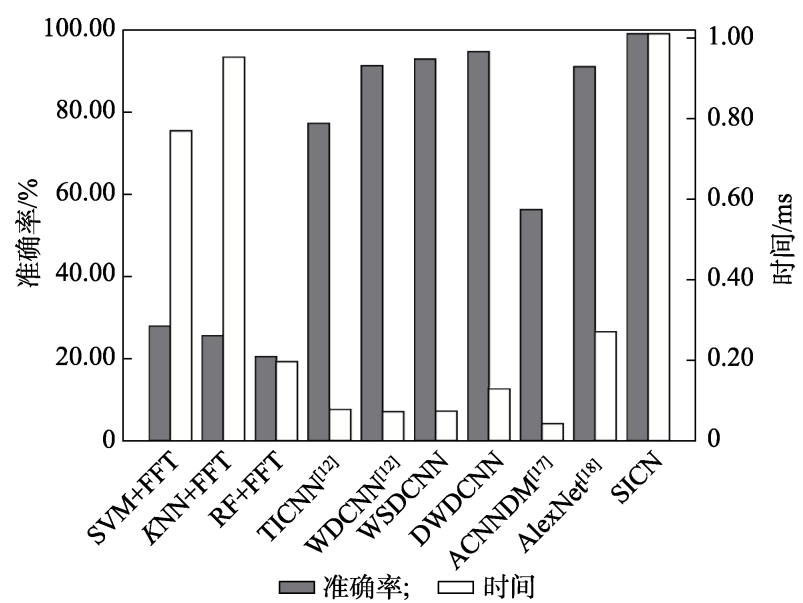

图 7 不同算法的准确率与时间性能对比

由图 7 可见，传统机器学习算法的表现相对较 差, 表现出低准确率，高时间消耗的特点，并不适 合用来诊断轴承故障. 而深度学习的几个模型中, ACNNDM 的时间消耗虽然最少，但其准确率相对 较低. WDCNN, WSDCNN, TICNN 的时间消耗相比 ACNNDM 略多一些，但准确率获得了极大的提高.

DWDCNN 虽然时间消耗长了一些，但其在准 确率上又有所提升, 且单条数据时间消耗仍不及 $0.20 \mathrm{~ms}$, 可以达到实时的要求. 相比之下, AlexNet 显然在准确率与时间消耗上均不如 DWDCNN 等. 而 SICN 虽然时间消耗较多，约 $1 \mathrm{~ms}$ 才能处理一条 数据, 但其准确率十分高, 显然适合对实时性要求 不高的情景.

\section{3 结 语}

本文针对轴承故障诊断的特点，提出了基于 
半实时的高准确率诊断方法. 本文 DWDCNN 比 WDCNN 等具有更高的准确率和鲁棒性，同时时 间性能相对较好，处理一条长度为 1920 的数据用 时仅为 $0.12 \mathrm{~ms}$. 本文的 SICN 具有更高的鲁棒性, 经实验验证, 在 $\mathrm{SNR}=-4 \mathrm{~dB}$ 的条件下依然可以达 到 $95.00 \%$ 的准确率，但由于该模型较大，处理一 条数据耗时约为 $1 \mathrm{~ms}$ ，因此更适合作为在极端环 境下或对不确定结果的二次诊断，或用于定期诊 断等对实时性要求不高的情景.

\section{参考文献(References):}

[1] National Manufacturing Power Construction Strategy Advisory Committee. "Made in China 2025" technical roadmap for key areas[OL]. [2020-08-16]. http://www.cae.cn/cae/html/files/ 2015-10/29/20151029105822561730637.pdf (in Chinese) (国家制造强国建设战略咨询委员会. 《中国制造 2025》重 点领域技术路线图 [OL]. [2020-8-16]. http://www.cae.cn/ cae/html/files/2015-10/29/20151029105822561730637.pdf)

[2] Tamilselvan P, Wang P F. Failure diagnosis using deep belief learning based health state classification[J]. Reliability Engineering \& System Safety, 2013, 115: 124-135

[3] Cortes C, Vapnik V. Support-vector networks[J]. Machine Learning, 1995, 20(3): 273-297

[4] Shao H D, Jiang H K, Wang F, et al. Rolling bearing fault diagnosis using adaptive deep belief network with dual-tree complex wavelet packet[J]. ISA Transactions, 2017, 69: 187-201

[5] Lu C, Wang Z Y, Qin W L, et al. Fault diagnosis of rotary machinery components using a stacked denoising autoencoderbased health state identification[J]. Signal Processing, 2017, 130: $377-388$

[6] Guo X J, Shen C Q, Chen L. Deep fault recognizer: an integrated model to denoise and extract features for fault diagnosis in rotating machinery[J]. Applied Sciences, 2017, 7(1): 41-42

[7] Hung Nguyen V, Sheng Cheng J, Yu Y, et al. An architecture of deep learning network based on ensemble empirical mode decomposition in precise identification of bearing vibration signal[J]. Journal of Mechanical Science and Technology, 2019, 33(1): 41-50

[8] Abed W, Sharma S, Sutton R, et al. A robust bearing fault detection and diagnosis technique for brushless DC motors under non-stationary operating conditions[J]. Journal of Control, Automation and Electrical Systems, 2015, 26(3): 241-254

[9] Pan H H, He X X, Tang S, et al. An improved bearing fault diagnosis method using one-dimensional CNN and LSTM[J]. Journal of Mechanical Engineering, 2018, 64(7/8): 443-452

[10] Qian W W, Li S M, Wang J R, et al. An intelligent fault diagnosis framework for raw vibration signals: adaptive overlapping convolutional neural network[J]. Measurement Science and Technology, 2018, 29(9): Article No.095009

[11] Hoang D T, Kang H J. Rolling element bearing fault diagnosis using convolutional neural network and vibration image[J]. Cognitive Systems Research, 2019, 53: 42-50

[12] Zhang Wei. Study on bearing fault diagnosis algorithm based on convolutional neural network[D]. Harbin: Harbin Institute of Technology, 2017(in Chinese) (张伟. 基于卷积神经网络的轴承故障诊断算法研究 [D]. 哈 尔滨: 哈尔滨工业大学, 2017)

[13] Sabour S, Frosst N, Hinton G E. Dynamic routing between capsules $[\mathrm{C}] / /$ Proceedings of the 31 st International Conference on Neural Information on Processing Systems. Cambridge: MIT Press, 2017: 3859-3869

[14] Zhu Zhiyu. Study on convolutional neural network based domain adaptive machine diagnosis methods[D]. Harbin: Harbin Institute of Technology, 2019(in Chinese)

(朱智宇. 基于卷积神经网络的机械故障诊断域自适应算法 研究[D]. 哈尔滨：哈尔滨工业大学, 2019)

[15] Szegedy C, Liu W, Jia Y Q, et al. Going deeper with convolutions[C] //Proceedings of the IEEE Conference on Computer Vision and Pattern Recognition. Los Alamitos: IEEE Computer Society Press, 2015: 1-9

[16] Lin T Y, Dollár P, Girshick R, et al. Feature pyramid networks for object detection[C] //Proceedings of the IEEE Conference on Computer Vision and Pattern Recognition Los Alamitos: IEEE Computer Society Press, 2017: 2117-2125

[17] Liu Xingchen, Zhou Qicai, Zhao Jiong, et al. Real-time and anti-noise fault diagnosis algorithm based on 1-D convolutional neural network[J]. Journal of Harbin Institute of Technology, 2019, 51(7): 89-95(in Chinese) (刘星辰, 周奇才, 赵昫, 等. 一维卷积神经网络实时抗噪故 障诊断算法[J]. 哈尔滨工业大学学报, 2019, 51(7): 89-95)

[18] Krizhevsky A, Sutskever I, Hinton G E. ImageNet classification with deep convolutional neural networks[C] //Proceedings of the 25th International Conference on Neural Information Processing Systems. Cambridge: MIT Press, 2012: 1097-1105 